\title{
Constraining gamma-ray burst progenitors
}

\author{
Andrew Levan \\ Department of Physics, University of Warwick, \\ Coventry, CV4 9GE, UK, \\ email: a.j.levan@warwick.ac.uk
}

\begin{abstract}
The past decade has seen great progress towards the unmasking of the progenitors of gamma-ray bursts, starting with the unambiguous detection of a supernova in the light of the long-GRB 030329 almost ten years ago, and the discovery of the first afterglows to shortGRBs in 2005. Here I review progress towards unveiling the progenitors of both long and shortduration GRBs. Furthermore, I examine the diverse broader population of GRBs and high energy transients, and suggest that a full consideration of this parameter space leads to the conclusion that additional progenitor models are likely to be needed, if we are to understand the complete view of GRBs and the transient high-energy sky.
\end{abstract}

Keywords. gamma-rays : bursts, (stars:) supernovae: general

\section{Introduction}

The search for the progenitors of gamma-ray bursts has long concentrated on unveiling the nature of two broad classes of gamma-ray bursts which have been known for more than twenty years, and are most cleanly separated by their durations and spectral hardness (Kouveliotou et al. 1993). The belief in this pursuit has generally been that each of these classes will ultimately be created by a single set of progenitors, or at least those which are very similar. In this sense, the goal of much GRB research has been to reduce the myriad of models for GRB production to only one or two canonical models.

This search has been extremely successful, we now believe that long-GRBs are created in massive star collapse (see Hjorth \& Bloom 2011 for a review). The short duration bursts remain more elusive, but the most promising model for their origin remains in the final merger of compact object binaries (e.g. NS-NS or NS-BH; for a review see Nakar et al. 2007). However, while this does indeed reduce the number of progenitor channels remarkably, there are many now disfavoured models that appear physically plausible, and therefore an important question is if any of these can still produce identifiable GRBs? Similarly, events over the past couple of years have shown surprising diversity in GRB populations. A crucial question is if this diversity is intrinsic to the progenitors creating GRBs, or is indicative of additional progenitor systems. In this contribution I will briefly discuss how we know the progenitors of GRBs, and how combinations of different techniques might ultimately allow us to isolate different possible progenitor models.

\section{Parameter Space}

In studies of optical transients many different classes of objects can be seen in a plot of luminosity against duration (e.g. Rau et al. 2009). These diverse events range from faint, fast novae to highly luminous and long-lived SNe, and represent a comparably diverse set of progenitor systems. Figure 1 shows a $\gamma$-ray equivalent of this plot, in which various "classes" of $\gamma$-ray transient have been marked (note that the borders between these are in many cases rather arbitrary, and in practice likely to be blurred). The classical 


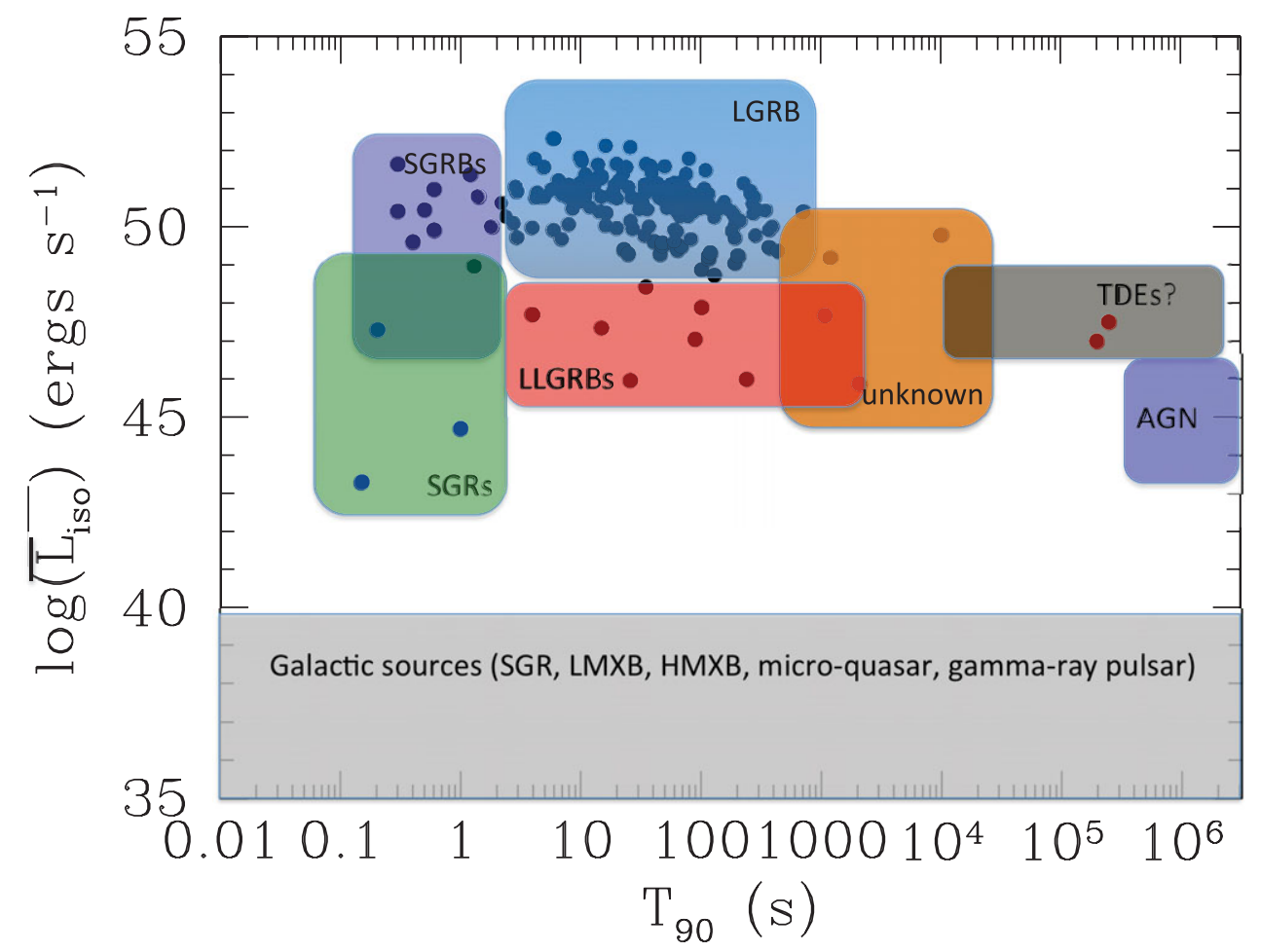

Figure 1. Gamma-ray transient parameter space, expressed as the duration over which $90 \%$ of the total fluence is measured $\left(T_{90}\right)$, and the average luminosity over this duration (note that the positions of the points within this plot are only approximate, given the differing energy regimes etc). The approximate regions in which various classes of transient are found are marked. Several of these in practice have significant overlap, and so the drawing of a boundary is challenging. A particular note of the last year is the discovery of numerous long duration transients, with durations from $\sim 1$ hour to several days.

long- and short-duration GRBs (LGRBs and SGRBs, respectively) can clearly be seen, but it is not yet clear if a single progenitor model is sufficient to adequately describe each class. Firstly, some Galactic soft-gamma-repeaters (SGRs) have exhibited flares of sufficient luminosity that they could be seen as SGRBs in external galaxies (Hurley et al. 2005 ) and it is likely that at least some of the SGRB population arise from this mechanism (Tanvir et al. 2005; Levan et al. 2008). Within the long-GRB distribution there is a population of low luminosity GRBs (LLGRBs) that have typically long durations, and are characterised by strong thermal emission. Aside from associated SN Ic (Pian et al. 2006; Starling et al. 2011) it is far from clear that these events should be considered as directly scaled down versions of the LGRBs population. Finally, a recently discovered population of transients with extremely long durations pushes the duration distribution of LGRBs even further, and it is not clear what (if any) connection these sources have to the LGRB population at large.

A full characterization of this parameter space is clearly needed, as is the case for the optical sky. Below I will consider some observational approaches that have been applied so far, focussing in particular on the better studied populations of "classical" LGRBs and SGRBs. 


\section{Observational Techniques}

\subsection{Spectroscopy}

Spectroscopic typing of supernovae associated with GRBs has become the gold-standard, and is generally viewed as the clinching proof that GRBs were associated with energetic type Ic supernovae (Hjorth et al. 2003; Stanek et al. 2003). GRB afterglows can crudely be modeled as power-laws in both time and frequency $F(\nu, t) \propto \nu^{\beta} t^{\alpha}$, where frequently $\alpha \sim$ $\beta \sim-1$. In contrast SN show broadly thermal emission (albeit often heavily modified), and rise slowly. Hence, as the GRB afterglow fades, it is possible to isolate the spectral signature of the $\mathrm{SN}$, as a change in the spectrum, whose template matches that of the class of broad lined SN Ic.

Spectroscopy provides by far the most detail about the SNe associated with GRBs, from detailed typing, to expansion velocities, to evidence for aspherical emission. However, the primary drawback is its limited redshift range, such detailed work is only possible for very low redshift $(z<0.2)$. Beyond this, it is possible to isolate SN signatures (e.g. Sparre et al. 2011), but even this is only possible to $z \sim 0.7-1$ (e.g. Della Valle et al. 2003). However, a potentially larger concern is that it is far from clear that the GRBs in the limited volume open to this technique are representative of GRBs as a class. Of the "best" examples, only GRB 030329 is a typical long duration GRB. The other nearby GRB/SN all show highly atypical prompt properties, often associated with long lived, low luminosity emission, probably belonging to the possibly distinct class of low-luminosity GRBs (LLGRBs; e.g. Campana et al. 2006; Pian et al. 2006; Starling et al. 2011). In this sense, one should be cautious drawing strong inferences from this spectroscopic evidence alone.

Similar strong spectroscopic signatures are not natural to isolate in SGRB progenitors. Spectroscopy of the afterglow may yield signatures of radioactive element production in so called macronovae, (e.g. Metzger \& Berger 2012) but such spectroscopy has yet to be successfully obtained. Ultimately spectroscopic observations probing the density of the interstellar (or intergalactic) medium around the SGRB may provide strong constraints on the SGRB environments, but a unambiguous signature is likely to be challenging.

\subsection{Photometry}

Photometric constrains on GRB progenitors largely come from the same principle as the spectroscopic ones. As the afterglow light fades, the fading is slowed, or even reversed by the rising of the supernova light. If the lightcurve is monitored in multiple colours this is normally accompanied by a pronounced reddening of the light (e.g. Zeh et al. 2004). Photometric evidence of SNe can be seen out to $z \sim 1$ and beyond (Zeh et al. 2004). From this it has been suggested that essentially all long duration GRBs are consistent with the association with a supernova. However, such work is itself difficult. It relies in the successful decomposition of afterglow + supernova + host galaxy. While the latter can be subtracted off (although this is not always done) the first two are complex, both afterglows and supernovae can show variations in brightness and rise/decay rates. Coupled with this additional complications such as refreshing of the afterglow shock, can even reverse the afterglow decay without an associated supernovae. However, the broad picture in which the majority of GRBs are associated with moderately luminous supernovae seems well justified.

For short GRBs, it seems likely that photometric studies are the place in which any macronova emission would be found. Firstly, such emission is likely to be faint, such that spectroscopic observations will be challenging. Secondly, the timescale of this emission is 
likely to be much more rapid than seen in supernovae, and so identifying a rising source, and obtaining subsequent spectroscopic follow-up will require a fast response.

\subsection{Host environments}

For more distant GRBs, or for an attempt to study the ensemble properties of a large sample, studies of the host galaxies can be extremely powerful. The host galaxies provide information about the stellar scale environments of the progenitors, and as such are a strong route to their identification. In particular, theoretical progress over the past several years suggests GRB progenitors arise from low metallicity progenitors (e.g. Langer et al. 2010), probably because magnetic breaking during mass loss slows the rotation of more metal rich progenitors too much for the formation of an accretion disc upon core collapse. This picture is supported from the bulk ensemble properties of GRB hosts, which appear to be smaller and less luminous than those of core collapse supernovae hosts at similar redshifts (Fruchter et al. 2006; Svensson et al. 2010). In the handful of cases where direct spectroscopic observations of the hosts have yielded reliable metallicity measurements the gas phase metallicities also appear to be low in comparison with the field (Modjaz et al. 2008; Levesque et al. 2010).

This paints a broadly supportive picture for the collapsar model. However, it should be noted that these insights arise largely (and sometimes exclusively) for bursts where precise positions are available, and these in turn typically come from a sample which exhibit bright optical afterglows. In these cases, extinction along the line of sight within the host galaxy is apparently low, and this may provide a significant bias to the host populations that the GRBs probe. More recent work, focussing on the nature of the host galaxies of dark-GRBs, where the optical afterglow is absent, probably because of heavy extinction in the host galaxy address these concerns. These studies show that the host galaxies of dark GRBs are systematically more luminous, and redder in colour than those of the optically bright host (e.g. Perley et al. 2009; Svensson et al. 2012). A natural explanation is that they are also more metal rich. It may be that the sightlines to regions of intensive star formation tend to be dust free only in the lower mass galaxies. If correct, and confirmed by further observations this could have important implications for the nature of GRB progenitors.

\subsection{Host locations}

It is not only the bulk properties of the hosts which provide diagnostics towards GRB progenitors. This can also be provided by the locations of GRBs within their host galaxies. LGRBs, because of the short lifetimes of their progenitors, would be expected to lie in the cores of star forming regions within their host galaxies. Indeed, this is what is seen, LGRBs are extremely concentrated on the light of their host galaxies, and are typically found at small radial offsets from their hosts (Bloom et al. 2002; Fruchter et al. 2006; Svensson et al. 2010). They are even more concentrated on their host light than corecollapse supernovae, and this is naturally explained by their production in more massive progenitors (Larsson et al. 2007; Raskin et al. 2008). However, while these results, and the comparisons they enable are extremely valuable, the fundamental information that can be derived from them is restricted by the resolution of the observations. Even with $H S T$ we can only probe regions several hundred parsecs across at typical GRB redshifts, and so tying the observed properties back to those of the progenitor stars is a challenging task.

While studies of the locations of LGRBs in their hosts have been extremely influential, they are pivotal for SGRBs, and may represent the best chance of identifying their progenitors prior to the advent of the next generation of gravitational wave detectors 
(in particular Advanced-LIGO and Virgo, expected in 2015; Abadie et al. 2010). If, as thought, SGRBs arise from compact object mergers, then the dynamics of the systems will scatter them well away from the host. Any binary undergoing a merger has survived two supernovae. Each of these SN is likely to have imparted a significant natal kick on the nascent neutron star, while mass loss in the binary at the time of the supernova produces an additional impulse which can propel the binary at several hundred $\mathrm{km} / \mathrm{s}$ (e.g. Church et al. 2011). The typical merger times for the binaries are poorly constrained but are likely to be $>10^{7}$ years in most cases (most known NS-NS binaries have merger times $>10^{8}$ years). Given this it is likely that SGRBs will be well scattered on their host galaxies, and may escape from low mass systems altogether.

These dynamics are very different from the bulk of the host galaxy stellar population; even intrinsically older systems (e.g white-dwarfs collapsing to neutron stars) would be expected to lie at locations in the host close to stars, the presence of a strongly kicked component would be strong evidence for the compact merger model.

In practice, observations of SGRBs broadly support this model. They are clearly scattered on their hosts, and are inconsistent with either tracing the LGRB distribution, or a linear relationship with the light, at high statistical significance (Fong et al. 2010).

\section{Oddballs}

Perhaps the most remarkable discoveries of the past several years have been of new bursts which don't fit naturally within the standard long/short paradigm. For example, the discovery in 2006 of two bursts (GRB 060505 and GRB 060614) with apparently long duration, but not associated with a supernovae can be taken as evidence that not all long duration GRBs create simultaneous SN (e.g. Fynbo et al. 2006; Della Valle et al. 2006; Gal-Yam et al. 2006). This may represent a new, previously unseen progenitor channel for GRBs, for example the direct collapse to a black hole (Fynbo et al. 2006), or could alternatively be indicative of the difficulties in isolating individual progenitor types within any given parameter space, such that these notionally long duration GRBs were in fact created from a progenitor akin to those of short GRBs (Gehrels et al. 2006). At the other end of the duration spectrum, gamma-ray transients of particularly long duration are also of great interest, and do not clearly fit within the natural paradigms. These in themselves are a diverse population, ranging in duration from a few hours, up to several days, and it is unclear what relation (if any) they have to the classical GRB population, whose duration distribution peaks at around a minute.

Perhaps most controversial is the burst of Christmas day 2010 (GRB 101225) and its apparent cousin GRB 111209A. These bursts exhibit gamma-ray emission for several hours, accompanied by bright, but apparently uncorrelated UV, and X-ray emission showing prominent dipping behaviour. The first of these two events lacks any redshift measurement, and its host galaxy is unresolved by HST (Tanvir et al. 2011). Given this, and its moderate Galactic latitude, it was suggested that it could be a Galactic source, perhaps caused by the disruption of a minor body around a neutron star (Campana et al. 2011) in a model with remarkable resonance with one initially proposed to describe GRBs prior to the afterglow revolution (e.g. Guseinov et al. 1974). A competing proposal is that the source is extragalactic, occurring inside a dense shell created at the latter stages of evolution in a binary progenitor (Thöne et al. 2011).

If indeed related then GRB 111209A offers a possible solution to these problems, since it was possible to obtain an absorption line redshift of $z=0.67$ (Vreeswijk et al. 2011), conclusively demonstrating an extragalactic origin. None-the-less, the true origin 
of these remarkably long bursts remains shrouded in mystery, and further studies of larger examples using the techniques described above are likely to prove highly diagnostic.

At the even more extreme end of the duration distribution is GRB 110328A (now known more commonly, and correctly, as Swift 1644+57). This "burst" was detected as a GRB, but had bright, long-lasting emission which clearly marks it apart from the bulk of this population. Its long lived, bright X-ray emission, coupled with an origin in the nucleus of an apparently inactive galaxy at $z=0.35$ suggest we may well be observing a relativistic jet formed at the time of tidal disruption of a star by the massive black hole in this galaxy (Levan et al. 2011; Bloom et al. 2011; Burrows et al. 2011; Zauderer et al. 2011). However, alternative explanations, with much closer resonance to standard LGRB production have also been proposed (Quataert \& Kasen 2012; Woosley \& Heger 2012).

\section{Conclusions}

Understanding the nature of GRB progenitors remains one of the central goals of high energy astrophysics. Despite the remarkable success of the past decade our understanding of the progenitors of long GRBs remains rudimentary, and the nature of short-GRBs is still plagued by uncertainty. Coupled with this, ongoing discoveries suggest that the high energy sky is much more diverse that we had previously appreciated. In particular, very long duration GRBs (which at some point possibly should not be called GRBs at all), may point the way to new progenitor channels. This may finally provide evidence for alternative routes that have been suggested for GRB creation, or even open up new, and so far unexplored channels for their production.

\section{References}

Abadie, J., Abbott, B. P., Abbott, R., et al. 2010, Classical and Quantum Gravity, 27, 173001

Bloom, J. S., Kulkarni, S. R., \& Djorgovski, S. G. 2002, AJ, 123, 1111

Bloom, J. S., Giannios, D., Metzger, B. D., et al. 2011, Science, 333, 203

Burrows, D. N., Kennea, J. A., Ghisellini, G., et al. 2011, Nature, 476, 421

Campana, S., Mangano, V., Blustin, A. J., et al. 2006, Nature, 442, 1008

Campana, S., Lodato, G., D'Avanzo, P., et al. 2011, Nature, 480, 69

Church, R. P., Levan, A. J., Davies, M. B., \& Tanvir, N. 2011, MNRAS, 413, 2004

Della Valle, M., Malesani, D., Benetti, S., et al. 2003, A\&SA, 406, L33

Della Valle, M., Chincarini, G., Panagia, N., et al. 2006, Nature, 444, 1050

Fruchter, A. S., Levan, A. J., Strolger, L., et al. 2006, Nature, 441, 463

Fong, W., Berger, E., \& Fox, D. B. 2010, ApJ, 708, 9

Fynbo, J. P. U., Watson, D., Thöne, C. C., et al. 2006, Nature, 444, 1047

Gal-Yam, A., Fox, D. B., Price, P. A., et al. 2006, Nature, 444, 1053

Gehrels, N., Norris, J. P., Barthelmy, S. D., et al. 2006, Nature, 444, 1044

Guseinov, O. K. \& Vanek, V. 1974, APSS, 28, L11

Hjorth, J., Sollerman, J., Møller, P., et al. 2003, Nature, 423, 847

Hjorth, J. \& Bloom, J. S. 2011, arXiv:1104.2274

Hurley, K., Boggs, S. E., Smith, D. M., et al. 2005, Nature, 434, 1098

Kouveliotou, C., Meegan, C. A., Fishman, G. J., et al. 1993, ApJL, 413, L101

Langer, N., van Marle, A.-J., \& Yoon, S.-C. 2010, New Astronomy Reviews, 54, 206

Larsson, J., Levan, A. J., Davies, M. B., \& Fruchter, A. S. 2007, MNRAS, 376, 1285

Levan, A. J., Tanvir, N. R., Jakobsson, P., et al. 2008, MNRAS, 384, 541

Levan, A. J., Tanvir, N. R., Cenko, S. B., et al. 2011, Science, 333, 199

Levesque, E. M., Kewley, L. J., Berger, E., \& Zahid, H. J. 2010, AJ, 140, 1557

Metzger, B. D. \& Berger, E. 2012, ApJ, 746, 48

Modjaz, M., Kewley, L., Kirshner, R. P., et al. 2008, AJ, 135, 1136 
Nakar, E. 2007, Physics Reports, 442, 166

Perley, D. A., Cenko, S. B., Bloom, J. S., et al. 2009, AJ, 138, 1690

Pian, E., Mazzali, P. A., Masetti, N., et al. 2006, Nature, 442, 1011

Quataert, E. \& Kasen, D. 2012, MNRAS, 419, L1

Raskin, C., Scannapieco, E., Rhoads, J., \& Della Valle, M. 2008, ApJ, 689, 358

Rau, A., Kulkarni, S. R., Law, N. M., et al. 2009, PASP, 121, 1334

Sparre, M., Sollerman, J., Fynbo, J. P. U., et al. 2011, ApJL, 735, L24

Stanek, K. Z., Matheson, T., Garnavich, P. M., et al. 2003, ApJL, 591, L17

Starling, R. L. C., Wiersema, K., Levan, A. J., et al. 2011, MNRAS, 411, 2792

Svensson, K. M., Levan, A. J., Tanvir, N. R., Fruchter, A. S., \& Strolger, L.-G. 2010, MNRAS, 405,57

Svensson, K. M., Levan, A. J., Tanvir, N. R., et al. 2012, MNRAS, 421, 25

Tanvir, N. R., Levan, A. J., Fynbo, J. P. U., et al. 2011, GRB Coordinates Network, 11564, 1

Thöne, C. C., de Ugarte Postigo, A., Fryer, C. L., et al. 2011, Nature, 480, 72

Vreeswijk, P., Fynbo, J., \& Melandri, A. 2011, GRB Coordinates Network, 12648, 1

Woosley, S. E. \& heger, A. 2011, arXiv:1110.3842

Zauderer, B. A., Berger, E., Soderberg, A. M., et al. 2011, Nature, 476, 425

Zeh, A., Klose, S., \& Hartmann, D. H. 2004, ApJ, 609, 952

\section{Discussion}

JeSPER Sollerman: Where next for short-GRBs?

ANDREW LEVAn: Short-GRBs are extremely challenging. The most obvious way to make progress is to get a coincident gravitational wave signal, but that is probably several years away (2015 for the first "light" of Advanced-LIGO), given this, the best way to make progress is i) to continue to build larger samples of SGRBs to study the locations of the bursts around their hosts, since this has significant statistical power and ii) to hope that through getting the best monitoring of these events that we can, that we might see an associated macronova.

EHUD NAKAR: How are you selecting your dark GRB redshifts (emission vs spectroscopic)

ANDREW LEVAN: This is a fair concern. A good fraction of optical bright LGRB redshifts come from the afterglow, and so are effectively independent of the host brightness. In contrast, if there is no optical afterglow to take a spectrum of, then we have to look for emission lines in the host galaxy. Although this is possible for very faint galaxies it can introduce a bias, in that it is easier to get such measurements for brighter galaxies, and this does somewhat impact the results that dark-GRB hosts are more luminous than the optically bright hosts. However the results seem to stand when considering this bias. 